\title{
Rapid Intubation Sequence: 4-Year Experience in an Emergency Department
}

\author{
Ángela María Muñoz $\mathbb{D}^{1,2}$ \\ Manuela Estrada (D) \\ Jaime A Quintero (D) ${ }^{1-3}$ \\ Mauricio Umaña (iD' \\ 'Emergency Department, Fundación Valle \\ del Lili, Cali, 760032, Colombia; \\ ${ }^{2}$ Universidad ICESI, Emergency Medicine \\ Residency, Cali, Colombia; ${ }^{3}$ Centro de \\ Investigaciones Clínicas (CIC), Fundación \\ Valle del Lili, Cali, 760032, Colombia
}

Correspondence: Jaime A Quintero; Mauricio Umaña

Email jaime.quintero.ra@fvl.org.co;

mauricio.umaña@fvl.org.co
Background: The rapid intubation sequence is advanced airway management that effectively ensures an adequate supply of oxygen in critically ill patients. The medical personnel in the emergency department performed this procedure.

Objective: To describe the main characteristics of the rapid intubation sequence in an emergency department of a high complexity hospital.

Methods: This is a descriptive, cross-sectional, retrospective study. We included all older patients with a rapid intubation sequence requirement in the emergency department from 2014 to 2017 . We used central tendency measures for numerical variables and proportions for categorical variables.

Results: A total of 401 patients were eligible for this analysis. The main indication for intubation was the Glasgow Coma Scale $=<8$ in 170 patients (42.4\%), followed by hypoxemia in 142 patients (35.4\%). In 36 patients, at least one complication occurred. RSI was performed in $54.4 \%$ by emergency physician. RSI was successful on the first attempt in $90.5 \%$. Only 36 patients (9\%) presented complications.

Conclusion: In this study, we found that the rapid intubation sequence was not related to a high proportion of complications. Perhaps, this is attributed to the degree of medical training and the use of emergency department protocols in our hospital.

Keywords: intubation, sequence, rapid intubation, emergencies, RSI

\section{Introduction}

The rapid intubation sequence (RSI) is an advanced technique in the management of airways that safely ensures an adequate supply of oxygen to critically ill patients in the emergency department. This procedure has proven to be useful in the approach of patients with a compromised state of consciousness or by alterations in respiratory dynamics treated in the emergency department. $^{1,2}$

The emergency department is the first scenery to determine a quick and concrete clinical assessment in the patient with the imminence of ventilatory failure, allowing to identify the type of airway according to the characteristics of the $^{\text {patient. }^{3-5}}$

The administration of an inducer and a relaxant are used simultaneously after placement of the orotracheal tube, being the most commonly used method in approximately $85 \%$ of intubations in the emergency room. ${ }^{6,7}$ The most appropriate choice of premedication improves the probability of success of orotracheal intubation by minimizing the risk of complications such as hypoxemia, cardiovascular complications, or other secondary events of the medication. ${ }^{8-10}$ 
Some studies realized in the emergency departments around the world have shown the experiences with the RSI. ${ }^{4,11,12}$ However, some research has certain limitations in determining the validity of their use in hospital environments. $^{13,14}$

Some authors have described the effectiveness in the first attempt compared to those in which RSI is not performed. ${ }^{4,13,15}$ Despite being a frequent procedure in emergency room, some complications that this procedure entails have been described. ${ }^{16,17}$

Previous studies in our region have not been developed to evaluate the rapid intubation procedure. Few intubation schemes have been implemented depending on the needs of the emergency room of each institution. However, a detailed description of the characteristics of the implementation and use of the RSI in different emergency rooms is necessary.

The objective of our study is to describe the main characteristics of the rapid intubation sequence used in patients treated in the emergency department of a high complexity hospital.

\section{Methods}

\section{Source of Data}

This retrospective descriptive study was conducted at Fundación Valle del Lili (FVL) University Hospital. The hospital is a non-profit university hospital affiliated with the Icesi University School of Medicine in Cali, Colombia. It is a referral hospital from the Southwest region of the country and has an emergency department volume of approximately 70,000 patients per year.

The ethics committee in biomedical research at VFL approved this study (Register No. 262-2019, approved on July 31, 2019. Institutional act No. 15 of July 31, 2019, protocol number 1421 and followed the Declaration of Helsinki Ethical Principles for Medical Research Involving Human Subjects). This study adhered to the standards of the STROBE guidelines (STROBE statement is provided in Supplementary Material).

This study did not present risk (according to resolution 8430 of 1993 of the Colombian Ministry of Health); since it is documentary in nature, and the researchers do not assign biological, psychological, or social exposures to patients. Therefore, the ethics committee omitted the use of informed consent. All patient data accessed complied with relevant data protection and privacy regulations.

\section{Patients}

The registry searched for data of patients admitted to the emergency department from 2015 to 2019 . We included patients older than 18 years treated in the emergency department who required advanced airway management with a rapid intubation sequence. We excluded patients intubated without RSI medications (analgesic, sedative, and muscle relaxant) and the pregnant patients.

Demographics and clinical variables, medications used in the rapid intubation sequence, medical experience, and complications presented during the procedure were collected. Complications were defined as any medical problem that occurred during the RSI.

\section{Statistical Analysis}

The normality of the variables was determined through a Shapiro Wilk test. A descriptive analysis was performed. The qualitative variables were presented using measures of relative and absolute frequencies. All frequencies were calculated as proportions, taking the events of interest as numerators and the studied population as the denominator. The quantitative variables were expressed through the measure of central tendency and dispersion, according to their distribution.

\section{Results}

Four hundred and one patients were included in the final analysis. The majority was male (61.4\%) and the mean age of patients was 55 years (IR 32-71). The most common comorbidities were hypertension (35.9\%) and diabetes $16 \%$ of the population.

The main indication for RSI was Glasgow $\leq 8$ in $42.5 \%$ (n: 170). In this population with Glasgow $\leq 8$ the $46.5 \%$ (n: 79) patients suffer a traumatic brain injury. The second most frequent indication was hypoxemia in $35.4 \%$ (n: 142) (Table 1. Demographic and clinical characteristics).

The most frequently used analgesic was fentanyl. Midazolam was the most frequently used sedative agent. In some patients, two sedative agents were used. The most frequently used paralytic agent was rocuronium. RSI was performed in $54.4 \%$ by emergency physician, followed by general practitioners in $30 \%$ and only $1.3 \%$ by emergency medicine resident. RSI was successful on the first attempt in $90.5 \%$ (Table 2. RSI medications and total if attempts).

Only 36 patients $(9 \%)$ presented complications, and 7 patients $(1.8 \%)$ in this group presented two complications. The most common comorbidities presented in this group 
Table I Demographic and Clinical Characteristics

\begin{tabular}{|l|c|}
\hline Sex, $\mathrm{n}(\%)$ & \\
Women & $155(38.7)$ \\
Man & $246(61.4)$ \\
\hline Age** & $55(32-71)$ \\
\hline Origin, $\mathrm{n}$ (\%) & \\
House & $136(33.9)$ \\
Hospital & $175(43.6)$ \\
Rural Area & $46(11.5)$ \\
Public roads & $44(11)$ \\
\hline Comorbidities, $\mathrm{n}$ (\%) & \\
Hypertension & $144(35.9)$ \\
Diabetes Mellitus & $64(16)$ \\
Coronary disease & $4 I(10.2)$ \\
Neurocardiovascular disease & $24(6)$ \\
Immuno-rheumatic disease & $8(2)$ \\
Oncological disease & $28(7)$ \\
\hline Indication of RSI, $\mathrm{n}$ (\%) & \\
Glasgow <8 & $170(42.4)$ \\
Hypoxemia & $142(35.4)$ \\
Neurological impairment & $120(30)$ \\
Traumatic brain injury & $104(25.9)$ \\
Induced unconsciousness (medical coma) & $60(15)$ \\
Ischemic or hemorrhagic stroke & $4 I(10.2)$ \\
Septic shock & $22(5.5)$ \\
Airway obstruction & $19(4.7)$ \\
Convulsions & $13(3.2)$ \\
Severe asthma crisis & $5(1.3)$ \\
Overdose & $5(1.3)$ \\
Airway burn & $3(0.8)$ \\
Hypercapnia & $1(0.3)$ \\
\hline
\end{tabular}

Note:**= Median (IQR).

were hypotension (41.7\%), cardiorespiratory arrest (36.1\%), and prolonged intubation (19.4\%). Seventy-five percent (n: 27) of individuals with hypotension, were older than 55 years and $60 \%$ received midazolam and $40 \%$ received propofol (Table 3 . Total of complications and Table 4. Level of physician training vs complications.).
Table 2 RSI Medications and Total of Attempts

\begin{tabular}{|l|l|}
\hline Analgesic Agent, $\mathbf{n}$ (\%) & $295(73.6)$ \\
Fentanyl & $50(12.5)$ \\
Ketamine & $51(12.7)$ \\
Fentanyl and Ketamine & $1(0.2)$ \\
Morphine & $4(1)$ \\
No analgesic & \\
\hline Sedative Agent, $\mathbf{n}(\%)$ & $190(47.4)$ \\
Midazolam & $93(23.2)$ \\
Propofol & $67(16.7)$ \\
Midazolam and Propofol & $38(9.5)$ \\
Ketamine & $9(2.2)$ \\
Midazolam and Ketamine & $3(0.7)$ \\
Thiopental & $1(0.2)$ \\
Midazolam and Thiopental & \\
\hline Paralytic Agent, $\mathbf{n}$ (\%) & $206(51.4)$ \\
Rocuronium & $106(26.4)$ \\
Succinylcholine & $89(22.2)$ \\
Vecuronium & \\
\hline RSI Attempts, $\mathbf{n}$ (\%) & $363(90.5)$ \\
I & $17(4.2)$ \\
2 & $21(5.2)$ \\
\hline 33 & \\
\hline
\end{tabular}

\section{Discussion}

In our study, the complications occurred in a low percentage. In the search conducted by Okubo et al, ${ }^{2}$ the most relevant results showed that the complication rates did not differ significantly between the groups with RSI and without RSI (12 vs 13\%; P = 0.59). Our results showed that the percentage of complications was much lower, possibly attributed to a better performance in the RSI. The complication rate in the group of emergency physicians was high. Perhaps, this group had many procedures performed compared to the group of general physicians. In addition, emergency physicians attended directly to patients with difficult airways and more critical conditions.

Table 3 Total of Complications

\begin{tabular}{|c|c|c|c|c|c|c|c|}
\hline Age Range & $\begin{array}{l}\text { Hypotension, } \\
\text { n: } 15 \text { (4I.7\%) }\end{array}$ & $\begin{array}{c}\text { Cardiac } \\
\text { Arrest, } \\
\mathrm{n}: 13 \\
(36.1 \%)\end{array}$ & $\begin{array}{l}\text { Prolonged } \\
\text { Intubation, } \\
\text { n: } 7 \text { (19.4\%) }\end{array}$ & $\begin{array}{c}\text { Oxygen } \\
\text { Desaturation, } \\
\text { n: } 5(13.9 \%)\end{array}$ & $\begin{array}{c}\text { Arrhythmia, } \\
\text { n: } 2(5.6 \%)\end{array}$ & $\begin{array}{l}\text { Esophageal } \\
\text { Intubation, } \\
\text { n: I (2.8\%) }\end{array}$ & $\begin{array}{c}\text { Total Patients } \\
\text { with } \\
\text { Complications, } \\
\text { n: } 36(100 \%)\end{array}$ \\
\hline Young adults (18-39) & I (6,7\%) & I (7.7\%) & I (I4.3\%) & $3(40 \%)$ & $0(0 \%)$ & I (100\%) & $5(13.9 \%)$ \\
\hline $\begin{array}{l}\text { Middle-aged adults } \\
(40-59)\end{array}$ & $5(33.3 \%)$ & $4(30.8 \%)$ & $0(0 \%)$ & $2(20 \%)$ & I (50\%) & $0(0 \%)$ & $8(22.2 \%)$ \\
\hline Old adults $(\geq 60)$ & $9(60 \%)$ & 8 (6I.5\%) & 6 (85.7\%) & $2(40 \%)$ & I (50\%) & $0(0 \%)$ & 23 (63.9\%) \\
\hline
\end{tabular}


Table 4 Level of Physician Training vs Complications

\begin{tabular}{|l|c|c|c|c|c|c|}
\hline $\begin{array}{l}\text { Presence of } \\
\text { Complication }\end{array}$ & $\begin{array}{c}\text { Emergency } \\
\text { Physician, n: 218 }\end{array}$ & $\begin{array}{c}\text { General Emergency } \\
\text { Physician, n: I 22 }\end{array}$ & $\begin{array}{c}\text { Other } \\
\text { Specialties, } \\
\text { n: 45 }\end{array}$ & $\begin{array}{c}\text { Specialist in Second } \\
\text { Specialty, n: I I }\end{array}$ & $\begin{array}{c}\text { Emergency } \\
\text { Medicine Resident, } \\
\text { n: 5 }\end{array}$ & $\begin{array}{c}\text { Total } \\
\text { No }\end{array}$ \\
Yes & $198(90.8)$ & $114(93.4)$ & $39(86.7)$ & $10(90.9)$ & $4(80)$ & $365(91)$ \\
I & $8(6.6)$ & $6(13.3)$ & $(9.1)$ & $36(9)$ \\
\hline
\end{tabular}

The most frequent complication in our patients was hypotension. Some studies have evaluated which factors are related to the presence of hypotension. Heffner et $\mathrm{al}^{18}$ shows that having a severity index of 0.9 is the most sensitive predictor for post intubation hypotension, in addition to other variables with CKD, advanced age, and the indication of intubation due to ventilatory failure. Smischney et al $^{19}$ proposes that having a mean arterial pressure below $5 \mathrm{mmHg}$ at normal, an advanced age, the use of neuro blocker, or suffering a complication during intubation predict the presence of hypotension. In our study, we did not perform previous measurements of blood pressure that could support this finding. Of the medications used in induction, most of the population received midazolam and not propofol. The evidence had described that these drugs produce hypotension, especially in those with significant bleeding, ${ }^{20}$ with propofol being the most related. ${ }^{21}$ This point could be an important reason why this outcome occurred. In our population, we found that $93.3 \%$ of these patients were above 45 years old, maybe the age could be another cause for this outcome.

Cardiac arrest was the second most frequent complication. A study conducted by Heffner et $\mathrm{al}^{22}$ showed that in those who underwent RSI, $4.2 \%$ had cardiac arrest, and its appearance was directly related to the severity index before intubation in addition to the hemodynamic status and previous oxygenation.

Another important outcome was the high success of RSI in the first attempt. This high rate of effectiveness is related to the degree of expertise of the medical team and the application of a standardized institutional guide in the management of the airway. Our results are similar to a study conducted by Sakles et $a 1,{ }^{23}$ who implemented a program to improve the quality of RSI in emergency medicine residents. Performed in 10 years, they went from a success of intubation in the first attempt from $73.1 \%$ (n: 342 in 2008) to $92.4 \%$ (n: 410 in 2017). Another study conducted by Okubo et $\mathrm{al}^{2}$ compared the effectiveness of RSI in a population of 2365 . With 761 patients intubated with RSI versus 1604 intubated without RSI, the effectiveness rate in the first inmate for the group with RSI was $73 \%$ and $63 \%$ in the group without RSI. In another study by Yoon et al, ${ }^{24}$ they had $88 \%$ intubation success on the first attempt. Other authors had described a success rate below $87 \%$. ${ }^{4,13,25,26}$

However, in our study, some patients required 3 or more attempts due to a difficult airway. When faced with a difficult airway without success on the first attempt and instead of repeating the standard approach and increasing complications, the physician should consider alternative extraglottic airway devices in patients with a difficult airway (the laryngeal mask, the i-gel, Air-Q, the Aura-I, Aura-Gain, and the esophageal-tracheal combitube). ${ }^{27}$ Despite technological advances and the development of new devices for airway management, RSI remains the cornerstone of care in the airway definitive.

The intubation management guidelines state that 3 is the maximum number of intubation attempts. ${ }^{28}$ When intubation failed is necessary to employ a different device or operator. In our study, after the three attempts, someone with a higher degree of training (anesthesiologist or emergency physician) performed the intubation. The specialist did not consider the use of an extraglottic device in the clinical approach. All patients necessitated a definitive airway secondary to the clinical condition.

As mentioned above, patients with traumatic brain injury are sensitive to changes in hemodynamic parameters, and adverse events can occur with the inadequate administration of medications. However, in our results, the patients with a traumatic brain injury did not present any hemodynamic complications. This result may be related to the adhesion of international protocols and the knowledge of the implications of the RSI medications.

The RSI is performed commonly by anesthesiologists who are responsible for managing the airway in surgical spaces and even in an emergency department. Klucka et $\mathrm{al}^{29}$ conducted survey among members of European Society of Anesthesiologist (ESA). A total of 1921 
anesthesiologists participated in the surveys. They considered that the risk of regurgitation or aspiration was the principal indication for the use of RSI in $94.6 \%$. The second cause was the trauma in $92.8 \%$. In comparison with our results, the emergency physician performed most of the RSI. Concerning the indication of RSI, there may be a relationship with our study, perhaps attributed to the risk of aspiration in patients with decreased Glasgow. The emergency physicians were responsible for the most RSI with an adequate response of success in intubation and a low frequency of complications.

As mentioned above, patients with traumatic brain injury are highly susceptible to changes in hemodynamic parameters and, adverse events can occur with inadequate administration of medications. However, in our results, these subjects with a traumatic brain injury did not present any hemodynamic complications. This result may be related to the adhesion of international protocols and the knowledge of the implications in the use of some RSI medications.

Opioids have been used in the rapid intubation sequence to reduce the cardiovascular response to laryngoscopy and they can also reduce the dose of the inducing agent, although these are not part of the classical technique and usually the opioids are used as analgesics. ${ }^{30,31}$ In our study, fentanyl was the most analgesic used in the management. Fentanyl reduces release of stress hormones, oxygen consumption, cerebral blood flow, and intracranial pressure. All this benefits critically ill patients and attenuates the sympathetic response induced by the laryngoscopy procedure. ${ }^{32}$ Recently, a survey was conducted with 1921 physicians of the European Society of Anesthesiology on rapid sequence induction, concluding that $65 \%$ used opioids during the initiation of anesthesia. The use of analgesics reduces the cardiovascular response to laryngoscopy and the dose of the induction agent. ${ }^{29}$

The most frequent sedative agent used in our study was Midazolam. It is a sedative agent with a faster effect due to its fat solubility and the highest availability of the benzodiazepine class. Nevertheless, hypotension and respiratory depression are common complications in hypovolemic patients. The survey previously concluded that the preferred choice of sedative induction agents in hemodynamically stable patients was propofol in $90.6 \%$ for adults with hemodynamic instability or at risk of hypotension in $42 \%{ }^{29}$ In another survey on the practice of RSI, 266 health professionals in the United Kingdom showed that $64 \%$ used propofol instead of thiopental, which may be due to the efficacy of propofol, and 160 of the 266 consultants used fentanyl as an opioid. ${ }^{33}$
The main strength of this study was the first attempt intubation success with a low percentage of complications. This outcome may be related to the expertise of the medical team and the adequacy of an RSI protocol.

During their training, the emergency medicine residents undergo training for two months in the surgery room with the anesthesiology team to develop skills in airway management. In addition, during the three years of training, they are exposed to critical patients in the resuscitation service in a highly complex institution. This standardized process, validated by the emergency department, anesthesiology service, and surgery departments of our institution, has made it possible to minimize possible operational complications in the intubation technique.

\section{Limitations}

Actually, the emergency resident performs the RSI with the assistance of a respiratory therapist and the supervision of an emergency physician, who intervenes in the procedure when necessary immediately. Benedetto et al reported that the experience of the intubating physician did not influence the complication rate of urgent intubation. ${ }^{34}$ On the contrary, Tayal et al concluded that the success of RSI by emergency medicine residents increased with experience. ${ }^{35}$ Another study conducted by Ulrich et $\mathrm{al}^{36}$ found that the first-year anesthesia residents and secondyear residents had no difference in complication rate.

The low participation of emergency medicine residents was a weakness in our study. In our hospital, the emergency medicine program opened in 2014 with low numbers of residents (2 students in a year), and the reason why the general physicians who performed the procedure had high participation in RSI. The airway specialists, the physiotherapist, and the respiratory therapist team supervise the performance in RSI.

Other weaknesses of this study were the absence of some recollection variables (physiological variables before the procedure, the dose used for each drug, mortality during and after SIR, and even before discharge from hospital, among others). For future studies, we propose to compare the group of emergency medicine resident to consolidate skills and knowledge in emergencies.

\section{Conclusion}

We found that the rapid intubation sequence was not related to a high proportion of complications. Perhaps, this is attributed to the degree of medical training and the use of emergency department protocols in our hospital. 


\section{Author Contributions}

All authors contributed to data analysis, drafting or revising the article, gave final approval of the version to be published, agreed to the submitted journal, and agree to be accountable for all aspects of the work.

\section{Funding}

This research did not receive any specific grant from funding agencies in the public, commercial, or not-forprofit sectors.

\section{Disclosure}

Angela Maria Muñoz, Manuela Estrada, Jaime A. Quintero, and Mauricio Umaña declare that we have no conflicts of interest for this work.

\section{References}

1. Driver BE, Klein LR, Prekker ME, et al. Drug order in rapid sequence intubation. Acad Emerg Med. 2019;26(9):1014-1021. doi:10.1111/acem.13723

2. Okubo M, Gibo K, Hagiwara Y, Nakayama Y, Hasegawa K. The effectiveness of rapid sequence intubation (RSI) versus non-RSI in emergency department: an analysis of Multicenter Prospective Observational Study. Int J Emerg Med. 2017;10(1):1-9. doi:10. 1186/s12245-017-0129-8

3. Kim JH, Kim YM, Choi HJ, Je SM, Kim E. Factors associated with successful second and third intubation attempts in the ED. Am J Emerg Med. 2013;31(9):1376-1381. doi:10.1016/j.ajem.2013.06.018

4. Walls RM, Brown CA 3rd, Bair AE, Pallin DJ. Emergency airway management: a multi-center report of 8937 emergency department intubations. J Emerg Med. 2011;41(4):347-354. doi:10.1016/j. jemermed.2010.02.024

5. Brown CA 3rd, Bair AE, Pallin DJ, Walls RM. Techniques, success, and adverse events of emergency department adult intubations. Ann Emerg Med. 2014;65(4):363-370. doi:10.1016/j.annemergmed.2014.10.036

6. Takahashi J, Goto T, Okamoto H, Hagiwara Y, Watase H, Shiga T; Japanese Emergency Medicine Network Investigators. Association of fentanyl use in rapid sequence intubation with post-intubation hypotension. Am J Emerg Med. 2018;36(11):2044-2049. doi:10. 1016/j.ajem.2018.03.026

7. Sakles JC, Chiu S, Mosier J, et al. The importance of first pass success when performing orotracheal intubation in the emergency department. Acad Emerg Med. 2013;20(1):71-78.

8. Goto T, Gibo K, Hagiwara Y, et al. Multiple failed intubation attempts are associated with decreased success rates on the first rescue intubation in the emergency department: a retrospective analysis of multicentre observational data. Scand J Trauma Resusc Emerg Med. 2015;23(1):5. doi:10.1186/s13049-014-0085-8

9. Saldías F. Secuencia rápida de intubación en el servicio de urgencia [Rapid sequence of intubation in the emergency department]. Rev Chilena de Med Inten. 2015;30(1):23-32. Spanish.

10. Jaber S, Jung B, Corne P, et al. An intervention to decrease complications related to endotracheal intubation in the intensive care unit: a prospective, multiple-center study. Intensive Care Med. 2009;36(2):248-255.

11. Hasegawa K, Shigemitsu K, Hagiwara Y, et al. Association between repeated intubation attempts and adverse events in emergency departments: an analysis of a multicenter prospective observational study. Ann Emerg Med. 2012;60(6):749-754.e742.3. doi:10.1016/j. annemergmed.2012.04.005
12. Pallin DJ, Dwyer RC, Walls RM, et al. Techniques and trends, success rates, and adverse events in emergency department pediatric intubations: a report from the national emergency airway registry. Ann Emerg Med. 2016;67(5):610-615.e611. doi:10.1016/j. annemergmed.2015.12.006

13. Bozeman WP, Kleiner DM, Huggett V. A comparison of rapid-sequence intubation and etomidate-only intubation in the prehospital air medical setting. Prehosp Emerg Care. 2006;10(1):8-13. doi:10.1080/10903120500366854

14. Sagarin MJ, Chiang V, Sakles JC, et al. Rapid sequence intubation for pediatric airway management. Pediatr Emerg Care. 2002;18 (6):417-423. doi:10.1097/00006565-200212000-00004

15. Kim C, Kang HG, Lim TH, Choi BY, Shin YJ, Choi HJ. What factors affect the success rate of the first attempt at endotracheal intubation in emergency departments? Emerg Med J. 2013;30(11):888-892. doi:10.1136/emermed-2012-201708

16. Hayes AH, Breslin DS, Mirakhur RK, et al. Frequency of haemoglobin desaturation with the use of succinylcholine during rapid sequence induction of anaesthesia. Acta Anaesthesiol Scand. 2001;45(6):746-749. doi:10.1034/j.1399-6576.2001.045006746.x

17. Heier T, Feiner JR, Lin J, Brown R, Caldwell JE. Hemoglobin desaturation after succinylcholine-induced apnea: a study of the recovery of spontaneous ventilation in healthy volunteers. Anesthesiology. 2001;94 (5):754-759. doi:10.1097/00000542-200105000-00011

18. Heffner AC, Swords DS, Nussbaum ML, Kline JA, Jones AE. Predictors of the complication of post intubation hypotension during emergency airway management. J Crit Care. 2012;27(6):587-593. doi:10.1016/j.jcrc.2012.04.022

19. Smischney NJ, Demirci O, Diedrich DA, et al. Incidence of and risk factors for post-intubation hypotension in the critically ill. Med Sci Monit. 2016;22:346-355. doi:10.12659/MSM.895919

20. Murphy MF, Hung OR, Law JA. Tracheal intubation: tricks of the trade. Emerg Med Clin North Am. 2008;26(4):1001-1014. doi:10.1016/j.emc.2008.07.009

21. Brentjens TE, Warner DO. Pharmacology \& physiology in anesthetic practice, 4th edition. Anesthesiology. 2006;105(4):864. doi:10.1097/ 00000542-200610000-00052

22. Heffner AC, Swords DS, Neale MN, Jones AE. Incidence and factors associated with cardiac arrest complicating emergency airway management. Jul. 2013;84(11):1500-1504.

23. Sakles JC, Augustinovich CC, Patanwala AE, Pacheco GS, Mosier JM. Improvement in the safety of rapid sequence intubation in the emergency department with the use of an airway continuous quality improvement program. Western J Emerg Med. 2019;20 (4):610-618. doi:10.5811/westjem.2019.4.42343

24. Yoon U, Mojica J, Wiltshire M, et al. Emergent airway management outside of the operating room - a retrospective review of patient characteristics, complications and ICU stay. BMC Anesthesiol. 2019;19(1):220. doi:10.1186/s12871-019-0894-4

25. Stein C. Student paramedic rapid sequence intubation in Johannesburg, South Africa: a case series. Afr J Emerg Med. 2017;7(2):56-62. ISSN 2211-419X. doi:10.1016/j.afjem.2017.01.005

26. Simpson J, Munro PT, Graham CA. Rapid sequence intubation in the emergency department: 5 year trends. Emerg Med J. 2006;23 (1):54-56. doi:10.1136/emj.2004.019398

27. Butler KH, Clyne B. Management of the difficult airway: alternative airway techniques and adjuncts. Emerg Med Clin North Am. 2003;21 (2):259-289. doi:10.1016/S0733-8627(03)00007-5

28. Ahmad I, El-Boghdadly K, Bhagrath R, et al. Difficult airway society guidelines for awake tracheal intubation (ATI) in adults. Anaesthesia. 2020;75(4):509-528. doi:10.1111/anae.14904

29. Klucka J, Kosinova M, Zacharowski K, et al. Rapid sequence induction an international survey. Eur J Anaesthesiol. 2020;37(6):435-442.

30. Wallace C, McGuire B. Rapid sequence induction: its place in modern anaesthesia. Contin Educ Anaesth Crit Care Pain. 2014;14 (3):130-135. doi:10.1093/bjaceaccp/mkt047 
31. Koerber JP, Roberts GEW, Whitaker R, et al. Variation in rapid induction techniques: current practice in Wales. Anaesthesia 2009;64(1):54-59. doi:10.1111/j.1365-2044.2008.05681.x

32. Mc Kinlay J, Moss E. Pharmacology of drugs used in neuro anaesthesia. Best Pract Res Clin Anaesthesiol. 1999;13(4):499-510. doi:10.1053/bean.1999.0044

33. Sajayan A, Wicker J, Ungureanu N, Mendonca C, Kimani PK. Current practice of rapid sequence induction of anaesthesia in the UK - a national survey. Br J Anaesth. 2016;117(Suppl 1):i69-i74. doi:10.1093/bja/aew017

34. Benedetto WJ, Hess DR, Gettings E, et al. Intubación traqueal urgente en unidades hospitalarias generales: un estudio observacional [Tracheal intubation urgent care in general hospital units: an observational study]. J Clin Anesth. 2007;19(1):20-24. Spanish. doi:10.1016/ j.jclinane.2006.

05.018
35. Tayal VS, Riggs RW, Marx JA, Tomaszewski CA, Schneider RE Intubación de secuencia rápida en una residencia de medicina de emergencia: tasa de éxito y eventos adversos durante un período de dos años. Acad Emerg Med. 1999;6(1):31-37. doi:10.1111/j.15532712.1999.tb00091.x

36. Schmidt UH, Kumwilaisak K, Bittner E, George E, Hess D. Efectos de la supervisión por parte de los anestesiólogos asistentes sobre las complicaciones de la intubación traqueal de emergencia [Effects supervision by the assistant anesthesiologists on the complications of emergency tracheal intubation]. Anesthesiology. 2008;109(6):973-977. Spanish. doi:10.1097/ALN.0b013e31 $818 \mathrm{ddb} 90$

\section{Publish your work in this journal}

The Open Access Emergency Medicine is an international, peerreviewed, open access journal publishing original research, reports, editorials, reviews and commentaries on all aspects of emergency medicine. The manuscript management system is completely online and includes a very quick and fair peer-review system, which is all easy to use. Visit http://www.dovepress.com/testimonials.php to read real quotes from published authors. 